\title{
Vascular Medicine
}

http://vmj.sagepub.com/

Images in vascular medicine : Fibromuscular dysplasia

Thomas N Abahji, Federico Tató, Thomas Hilbertz, Hermann Berger and Ulrich Hoffmann

Vasc Med 2004 9: 311

DOI: $10.1191 / 1358863 \times 04 v m 555 x x$

The online version of this article can be found at:

http://vmj.sagepub.com/content/9/4/311

\author{
Published by: \\ (9)SAGE \\ http://www.sagepublications.com \\ On behalf of:

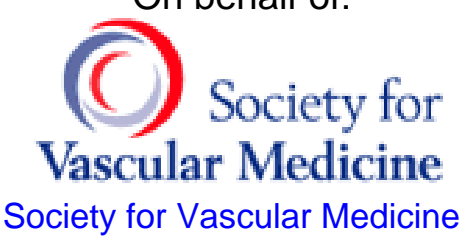

Additional services and information for Vascular Medicine can be found at:

Email Alerts: http://vmj.sagepub.com/cgi/alerts

Subscriptions: http://vmj.sagepub.com/subscriptions

Reprints: http://www.sagepub.com/journalsReprints.nav

Permissions: http://www.sagepub.com/journalsPermissions.nav

Citations: http://vmj.sagepub.com/content/9/4/311.refs.html

>> Version of Record - Nov 1, 2004

What is This? 


\section{Images in vascular medicine}

\section{Fibromuscular dysplasia}

\section{Thomas N Abahji ${ }^{a}$, Federico Tató ${ }^{a}$, Thomas Hilbertz ${ }^{b}$, Hermann Berger ${ }^{c}$ and Ulrich Hoffmann ${ }^{a}$}

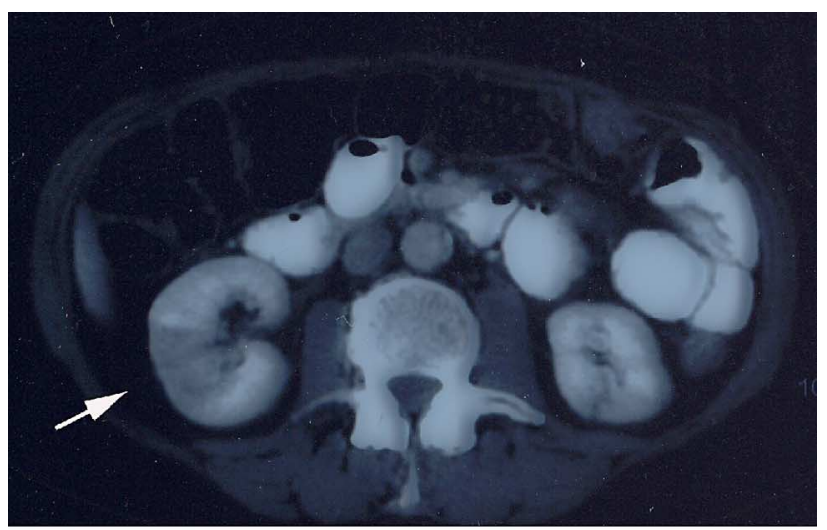

Panel A

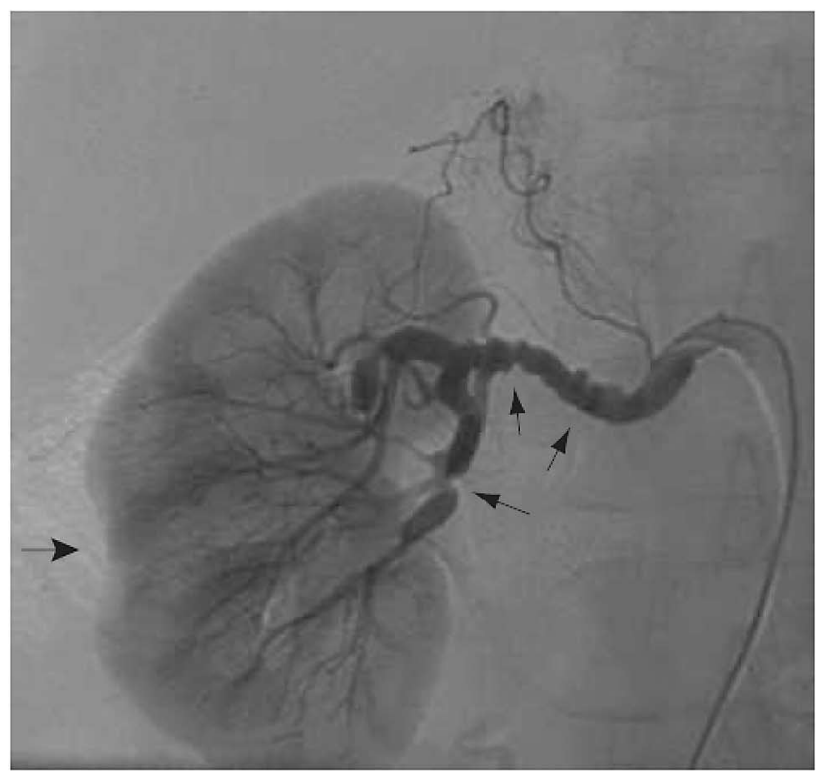

Panel B
A 60-year-old female presented with epigastric cramping and vomiting. Physical examination and initial laboratory work-up was unremarkable, except for severe abdominal tenderness and an elevated lactate dehydrogenase. A CT scan showed a hypodense, triangle-shaped area in the right kidney (Panel A), suggesting renal infarction. Renal duplex scanning revealed a focal increase of flow velocities followed by turbulence in multiple segments of the right renal artery. Arteriography showed multiple lesions ('string of beads') and a parenchymal filling defect (Panel B), establishing the diagnosis of fibromuscular dysplasia (FMD).

FMD most often affects young to middle-aged women (woman to man ratio $=3: 1$ ).$^{1}$ It is an arterial disease of unknown etiology typically involving the medium and large arteries. ${ }^{2}$ The renal and carotid arteries tend to be the most common location, but also other areas such as the iliac, mesenteric, or vertebral arteries may be affected. ${ }^{3}$ Histologically, an intimal, medial or subadventitial fibroplasia of the arterial wall can be seen. The medial type is most common and leads to luminal stenosis alternating with aneurysmal outpouchings. ${ }^{4}$ This results in the typical 'string of beads' appearance on arteriography, which is considered pathognomonic for FMD. ${ }^{5}$ The main complications of FMD may arise from dissection, emboli (as in this case) and aneurysm rupture. Diagnosis is established preferentially by arteriography. These typical findings may also be identified by MR arteriography and CT arteriography. With duplex ultrasound, typically a series of stenoses is detected, ${ }^{6}$ and is often the first diagnostic hint.

\section{References}

1 Safian RD, Textor SC. Medical progress: renal-artery stenosis. $N$ Engl $J$ Med 2001; 344: 431-42.

2 Sang CN, Whelton PK, Hamper UM et al. Etiologic factors in renovascular fibromuscular dysplasia. A case-control study. Hypertension. 1989; 14: $472-79$

3 Lüscher TF, Lie JT, Stanson A, Houser O, Hollier LH, Sheps SG. Arterial fibromuscular dysplasia. Mayo Clin Proc 1987; 62: 931-52.

4 Harrison EG. Pathologic classification of renal arterial disease in renovascular hypertension. Mayo Clin Proc 1971; 46: 161-67.

5 Kincaid OW, Davis GD, Hallermann FJ, Hunt JC. Fibromuscular dysplasia of the renal arteries: arteriographic features, classification, and observations on natural history of the disease. Am J Roentgenol 1968; 104: $271-82$.

6 Edwards JM, Zaccardi MJ, Strandness DE Jr. A preliminary study of the role of duplex scanning in defining the adequacy of treatment of patients with renal artery fibromuscular dysplasia. J Vasc Surg 1992; 15: 604-609.

\footnotetext{
a Division of Vascular Medicine, Ludwig-Maximilians University Hospital, Munich, Germany; ${ }^{b}$ Radiology Clinic, Prinzregentenplatz, Munich, Germany; ' Department of Interventional Radiology, Technical University, Munich, Germany

Address for correspondence: Ulrich Hoffmann, Division of Vascular Medicine, Medizinische Poliklinik, Ludwig-Maximilians University, Pettenkoferstr. 8a, 80336 Munich, Germany. E-mail: Ulrich.Hoffmann@med.uni-muenchen.de
} 
'Images in vascular medicine' is a regular feature of Vascular Medicine. Readers may submit original, unpublished images related to clinical vascular medicine to: Mark A Creager, Editor in Chief, Vascular Medicine, Brigham and Women's Hospital, 75 Francis Street, Boston, MA 02115, USA. 